\title{
Relación entre memoria secuencial auditiva, integración auditiva y desempeño fonológico en niños de 3 años a 3 años 11 meses con trastorno específico del lenguaje de tipo expresivo
}

\author{
Relationship between auditory sequential memory, \\ auditory integration and phonological performance in \\ children from 3 to 3 years 11 months with expressive \\ specific language impairment
}

\author{
Romina Arévalo \\ Universidad Católica de la Santísima \\ Concepción \\ Hernán León \\ Departamento de Fonoaudiología \\ Universidad de Concepción
}

\section{RESUMEN}

\begin{abstract}
La presente investigación tiene por objetivo estudiar la correlación entre las habilidades de memoria secuencial auditiva (MSA), integración auditiva (IA) y el desempeño fonológico en 24 niños con trastorno específico del lenguaje (TEL) expresivo de un rango etario de 3 años a 3 años 11 meses que asisten a una escuela especial de lenguaje. Los participantes fueron evaluados mediante la aplicación de dos subtest del Test Illinois de Aptitudes Psicolingüísticas (subtest de MSA y subtest de IA), y el Test para Evaluar los Procesos de Simplificación Fonológica Revisado (TEPROSIF-R). Los resultados muestran una correlación estadísticamente significativa entre memoria secuencial auditiva, integración auditiva y desempeño fonológico. Al analizar los datos dividiendo la muestra en dos grupos etarios se observa que, para el grupo etario de 3 años a 3 años 5 meses existe una correlación estadísticamente significativa entre MSA, IA y desempeño fonológico. En cambio, para el grupo etario de 3 años 6 meses a 3 años 11 meses se observa la misma tendencia sin alcanzar valores estadísticamente significativos. Esto sugiere que la relación entre estas variables disminuye a medida que los niños se acercan a su cuarto año de vida.
\end{abstract}

Palabras clave: trastorno especifico del lenguaje, proceso fonológico, integración auditiva, memoria secuencial auditiva.

\begin{abstract}
The present research aims to study the correlation between auditory sequential memory capacity (ASM), auditory integration (Al) abilities, and phonological performance in 24 children with expressive specific language impairment, with an age range between 3 and 3 years and 11 months, who attend a special language school. Participants were evaluated through two subtests of the Illinois Psycholinguistic Aptitudes Test (an ASM subtest and an Al subtest), and the Test to Evaluate the Phonological Simplification Processes Revised (TEPROSIF-R). The results indicate a statistically significant correlation between sequential auditory memory, auditory integration and phonological performance. When analyzing the data by dividing the sample into two age groups. When analyzing the data dividing the sample into two age groups, we observed that, for the age group between 3 and 3 years and 5 months there is a statistically significant correlation between auditory sequential memory, auditory integration and phonological performance. For the age group from 3 years 6 months to 3 years 11 months the same trend is observed without reaching statistically significant values. This suggests that the relationship between these variables decreases as children approach their fourth year of life.
\end{abstract}

Keywords: specific language impairment, phonological process, auditory integration, auditory sequential memory. 


\section{Introducción}

El Trastorno Específico del Lenguaje (TEL) ha sido descrito desde diferentes perspectivas teóricas. En la producción verbal de los niños con este diagnóstico se observan alteraciones que pueden presentarse con mayor o menor compromiso en los diferentes niveles de la lengua. Las dificultades del nivel fonológico son las de mayor frecuencia, constituyendo generalmente el signo más visible del trastorno y la principal causa de consulta de los padres.

Una perspectiva teórica que se ha usado para abordar este nivel es la Teoría de Fonología Natural (Stampe, 1969). Esta teoría reconoce en el niño la existencia de procesos de simplificación fonológica (PSF) que los niños utilizan al reproducir el habla adulta. Los PSF disminuyen progresivamente desde el inicio del desarrollo hasta la madurez fonológica, momento en que desaparecen. Así, un desarrollo fonológico apropiado se evidencia por una disminuida cantidad de PSF. Por el contrario, un menor desarrollo fonológico se manifiesta por una gran cantidad de PSF.

La teoría de la Fonología Natural permitió explicar los trastornos fonológicos. De este modo, Ingram (1983) aplicó dicha teoría para explicar los problemas fonológicos de los niños hablantes de inglés. Posteriormente, Bosch (2003) realizó lo mismo con niños hablantes de español peninsular.

En Chile se ha descrito desde esta perspectiva el desempeño fonológico de los niños con TEL (Pavez, 1990) y se ha elaborado un instrumento que mide PSF (Pavez, Maggiolo, y Coloma, 2008). A partir de los datos obtenidos con este instrumento, se han descritos perfiles de desarrollo fonológico típico para niños chilenos (Pavez, Maggiolo, Peñaloza, y Coloma, 2009). Ello ha posibilitado determinar patrones de producción fonológica en niños con trastorno fonológico (Pavez, Coloma, Maggiolo, y Peñaloza, 2013). Aun cuando se cuenta con información sobre las características del trastorno fonológico, se discute sobre las posibles causas que originan dificultades fonológicas en los niños con TEL.

Una disciplina que entrega interesantes explicaciones es la psicolingüística, la cual estudia diferentes habilidades cognitivas necesarias para el adecuado desarrollo del lenguaje. Es así como se señala que en niños con trastorno del lenguaje algunas habilidades cognitivas estarían descendidas. Al respecto, Triadó y Forns (1989) y Maurens, Olivares, y Vergara (2013) reportan que las habilidades psicolingüísticas más alteradas en niños con TEL son: (1) la integración auditiva (IA), que corresponde a la habilidad de analizar y sintetizar palabras y sílabas mediante la segmentación y el cierre fonológico (Kirk, MacCarthy, y Kirk, 2009) y (2) la memoria secuencial auditiva (MSA), que corresponde a la habilidad de repetir correctamente una secuencia de símbolos. Ello requiere almacenar y recordar información auditiva y verbal en el orden en que fue presentada (Kirk et al., 2009).

Cabe señalar que la evaluación de la IA en niños con TEL es recomendada por Carballo (2012) como complemento a la valoración del desempeño fonológico en una propuesta de protocolo de evaluación para el TEL. En cuanto a la MSA, diversos estudios detectan diferencias significativas al comparar niños con TEL con pares de niños con desarrollo típico (Blanco, González, Ramírez, Torres, y Valenzuela, 2008). 
En Chile, aún son escasas las investigaciones que vinculan el desarrollo lingüístico y cognitivo, sobre todo en las etapas iniciales del comportamiento oral. Ello, a pesar de que existe acuerdo sobre la importancia de su manejo para garantizar el éxito en tareas relevante, como es el aprendizaje. Todavía más escasa ha sido la investigación de estos aspectos en niños con un desarrollo atípico del lenguaje como en el caso del TEL, patología que afecta frecuentemente a pre-escolares y escolares de nuestro país.

Considerando lo expuesto, el presente estudio tiene por objetivo principal relacionar las habilidades cognitivas de IA y MSA con el desempeño fonológico en niños de 3 años con diagnóstico de TEL expresivo.

\section{Metodología}

\section{Participantes}

En la presente investigación se estudiaron las relaciones entre habilidades de MSA, IA y el desempeño fonológico en una muestra de 24 participantes con TEL expresivo que asisten a una escuela especial de lenguaje de la comuna de Quillón, Chile. Los rangos de edad estudiados fueron: de 3 años a 3 años 5 meses y de 3 años 6 meses a 3 años 11 meses, seleccionándose 12 niños para cada rango. La elección de los participantes se llevó a cabo de forma no probabilística, según las necesidades del estudio. Se cumplió con los siguientes criterios de inclusión: pertenecer a la Escuela Especial de Lenguaje de donde se obtiene la muestra, poseer entre 3 años y 3 años 11 meses de edad y presentar TEL expresivo, según la normativa y procedimiento que indica el Ministerio de Educación de Chile para realizar este diagnóstico. De igual forma se consideraron los siguientes criterios de exclusión: Presentar sospecha de otro diagnóstico y/o derivación por otras alteraciones.

\section{Procedimientos e instrumentos de evaluación}

La medición de las variables IA y MSA se realizó con dos sub-test del ITPA: a) MSA y b) IA.

a) MSA: La tarea consiste en pedir al niño que repita series de dígitos cada vez más largas, que se le van presentando a un ritmo uniforme, sin variar el tono de voz. Se comienza con el elemento número 1 en todos los casos y se detiene la aplicación al tercer fracaso consecutivo. Cada respuesta correcta se valora con un punto, siendo la puntuación máxima de 26.

b) IA: La tarea consiste en pedir al niño que complete una palabra que se le entrega incompleta y luego emitirla en su forma completa. Esta constituida por 29 estímulos y cada uno de ellos se valora con un punto.

La Tabla 1 presenta las medias y la desviación típica (DT) para el rango de edad de 3 años, expuestas en el ITPA para niños hablantes de español.

Tabla 1

Medias y DT para el rango de 3 años en ITPA.

\begin{tabular}{lcc}
\hline & Media & DT \\
\hline MSA & 4,24 & 2,23 \\
IA & 5,44 & 3,83 \\
\hline
\end{tabular}

La variable de desempeño fonológico se evaluó aplicando el "Test para Evaluar Procesos de 
Simplificación Fonológica" (TEPROSIF-R) (Pavez et al., 2008). Este test está validado en Chile y permite determinar la cantidad y los tipos de PSF que producen los niños. Consta de 36 ítems, donde se entrega un estímulo auditivo, acompañado de uno visual, para inducir la respuesta verbal del niño, a través de la imitación diferida. Ella consiste en que el niño tiene un tiempo entre la audición y producción de la palabra evaluada.

La Tabla 2 presenta los rendimientos correspondientes a la norma de los niños chilenos.

Tabla 2

Niveles de desempeño en TEPROSIF-R según el total de PSF de acuerdo con el rango de edad.

\begin{tabular}{lccc}
\hline Edad & \multicolumn{3}{c}{ Niveles de Desempeño } \\
\hline & Normal (No PSF) & Riesgo (No PSF) & Déficit (No PSF) \\
\cline { 2 - 3 } $3.0-3.11$ años & 0 a 42 (prom. 27) & 43 a 57 & 58 o más (3a D.E 72) \\
$4.0-4.11$ años & 0 a 23 (prom. 13) & 24 a 33 & más (3 D.E 43) \\
$5.0-5.11$ años & 0 a 14 (prom. 8) más (3a D.E 27) \\
$6.0-6.11$ años & 0 a 10 (prom. 5) & 15 a 21 & 16 o más (3a D.E 20) \\
\hline
\end{tabular}

\section{Análisis de los datos}

Una vez obtenidos los datos de las pruebas, se describe en primero el comportamiento de cada variable, para la totalidad de los sujetos y luego para cada rango etario por separado. Seguidamente, se realiza el análisis estadístico que entrega los valores de tendencia general. Luego, se aplica la prueba de coeficiente de correlación de Spearman y se establecen las relaciones entre las variables estudiadas. Así, se asociaron los puntajes de MSA con los puntajes del desempeño fonológico. El mismo procedimiento se efectuó entre los puntajes IA con los puntajes del TEPROSIF-R.

\section{Resultados}

\section{Desempeño en pruebas aplicadas}

Para el grupo de niños de 3 años a 3 años 5 meses, los resultados obtenidos en IA indican, que la media de los puntajes de los 12 participantes fue de 0,5 con una DT de 0,5. Como se aprecia en el Gráfico 1, este grupo presenta resultados con puntajes inferiores al de mayor edad, lo que obtuvieron una media de 1,5 con una DT de 0,8. 


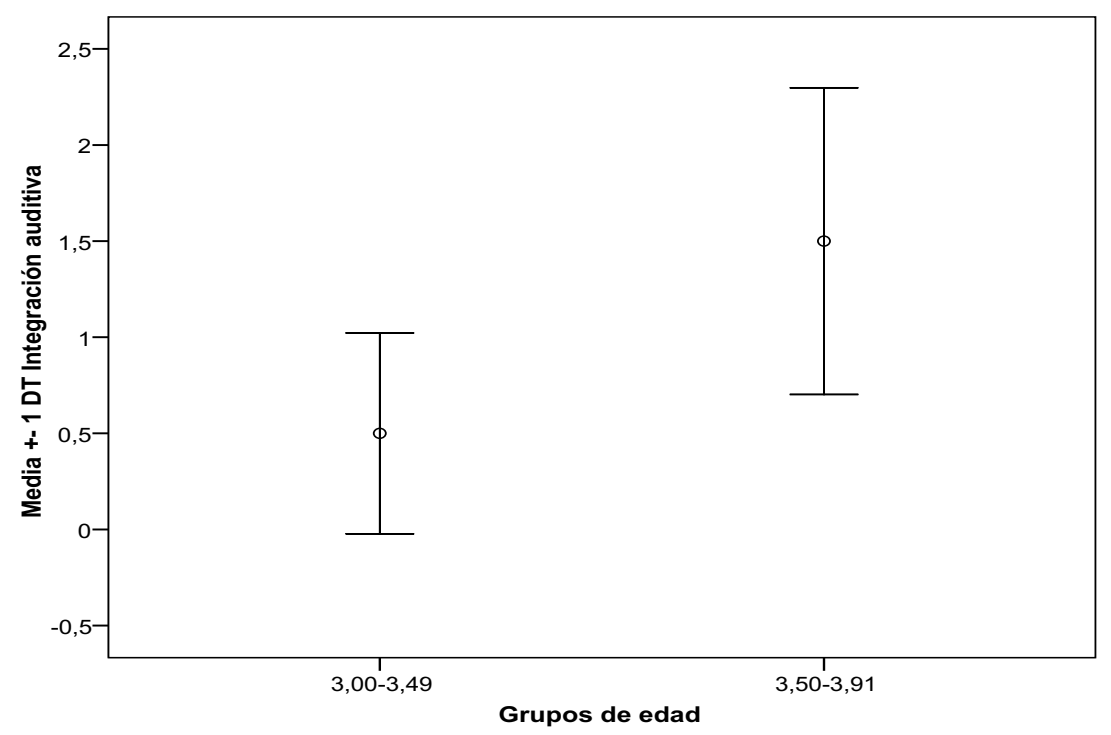

Gráfico 1. Puntaje de la Prueba de IA por grupo etario.

En la prueba de MSA, la media de los puntajes de los 12 participantes de menor edad fue de 1,3 con una DT de 0,8. La media del grupo de mayor edad alcanzó fue de 2,3 con una DT de 0,5. En el Gráfico 2 se advierte que los niños menores presentan medias inferiores a los participantes de mayor edad.

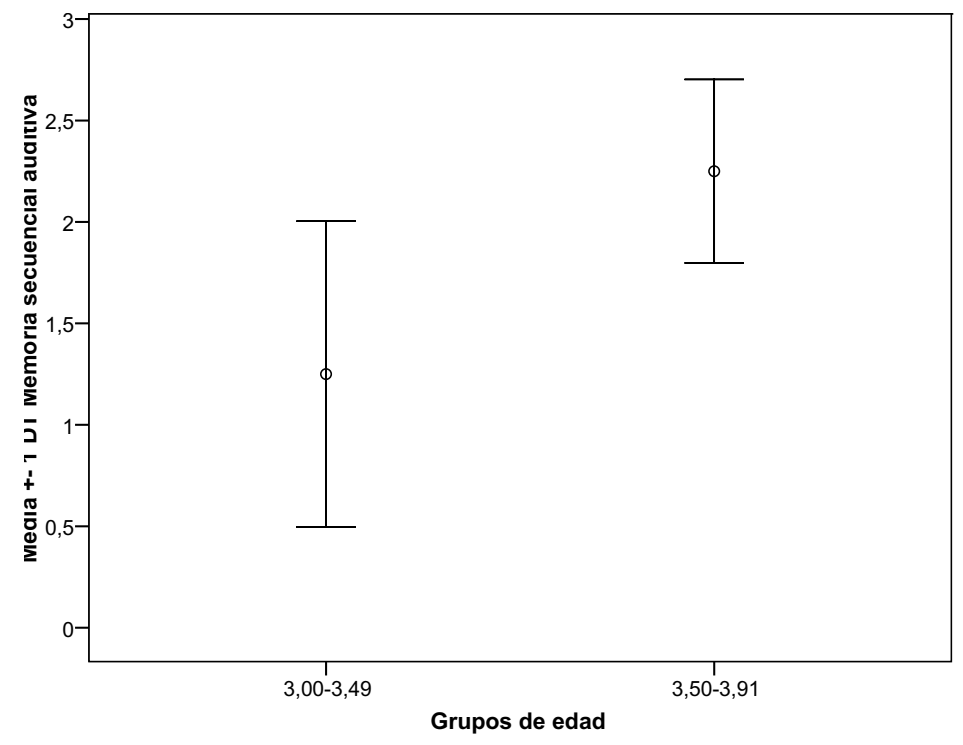

Gráfico 2. Puntaje de la Prueba de MSA por grupo etario. 
Relación entre memoria secuencial auditiva, integración auditiva y desempeño fonológico en niños de 3 años a 3 años 11 meses con trastorno específico del lenguaje de tipo expresivo

Los resultados obtenidos en el TEPROSIF-R indican que de los participantes de menor edad obtienen una media correspondiente a 76,5 puntos con una DT de 7,9. Por su parte, el grupo de niños mayores presenta una media de 62,4 puntos con una DT de 5,4. Es decir, los niños menores presentan más PSF que los de mayor edad. El Gráfico 3 ilustra estas diferencias.
Cabe destacar que, aunque los desempeños fonológicos son diferentes, ambos grupos se ubican en la categoría de déficit fonológico, según las normas del instrumento (ver Tabla 2).

A continuación, también se presenta la Tabla 3 que resume los estadísticos descriptivos de las pruebas aplicadas por grupo etario.

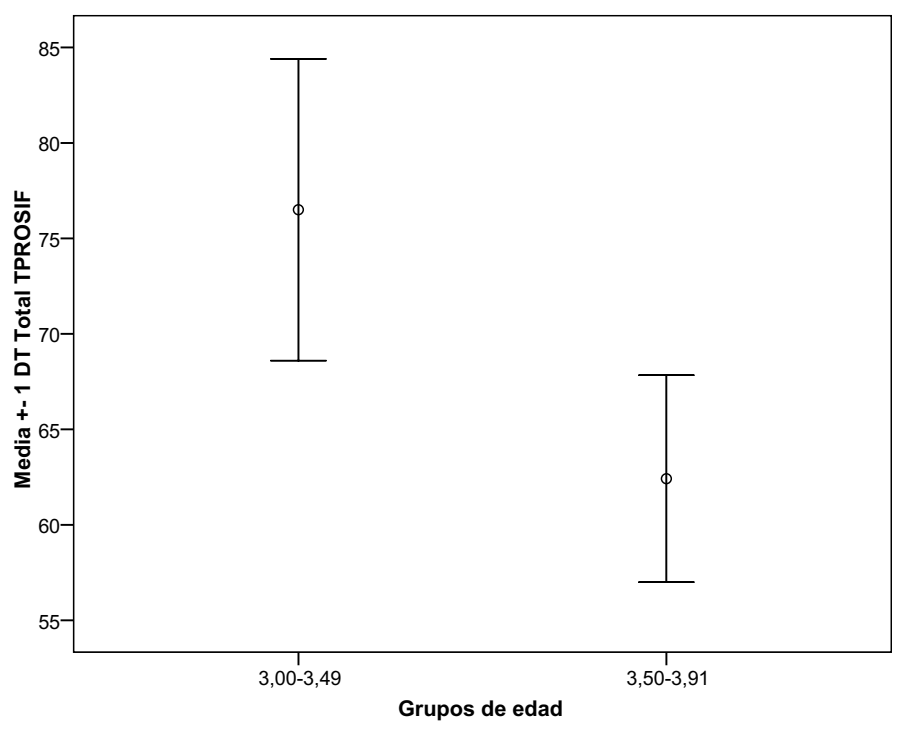

Gráfico 3. Puntaje de TEPROSIF-R Total por grupo etario.

Tabla 3

Estadísticos descriptivos de las pruebas aplicadas por grupo etario.

\begin{tabular}{|c|c|c|c|c|}
\hline Prueba & Grupos etarios & $\mathrm{N}$ & Media & DT \\
\hline \multirow[t]{2}{*}{ Integración auditiva (IA) } & $3,0-3,5$ & 12 & 0,5 & 0,5 \\
\hline & $3,6-3,11$ & 12 & 1,5 & 0,8 \\
\hline \multirow[t]{2}{*}{ Memoria secuencial auditiva (MSA) } & $3,0-3,5$ & 12 & 1,3 & 0,8 \\
\hline & $3,6-3,11$ & 12 & 2,3 & 0,5 \\
\hline \multirow[t]{2}{*}{ TEPROSIF-R } & $3,0-3,5$ & 12 & 76,5 & 7,9 \\
\hline & $3,6-3,11$ & 12 & 62,4 & 5,4 \\
\hline
\end{tabular}




\section{Análisis de correlación entre los puntajes de las pruebas psicolingüísticas y fonológica}

Se presenta el análisis de correlación entre los puntajes obtenidos en IA, MSA y TEPROSIF-R. En primer lugar para el total de los sujetos participantes se aprecia la existencia de una correlación bilateral significativa entre los resultados del TEPROSIF-R y la prueba de IA (ver Tabla 4). En síntesis, observamos en el Gráfico 4 que, al disminuir el puntaje obtenido en la prueba de IA, aumenta el número de PSF emitidos por los participantes en el TEPROSIF-R.

Tabla 4

Correlación entre TEPROSIF-R e IA.

\begin{tabular}{|c|c|c|c|c|}
\hline & & & TEPROSIF-R & IA \\
\hline \multirow[t]{6}{*}{ Rho de Spearman } & TEPROSIF-R & Coeficiente de correlación & 1,0 & $-0,6(* *)$ \\
\hline & & Sig. (bilateral) & . & 0,0 \\
\hline & & $\mathrm{N}$ & 24 & 24 \\
\hline & IA & Coeficiente de correlación & $-0,6(* *)$ & 1,0 \\
\hline & & Sig. (bilateral) & 0,0 & . \\
\hline & & $\mathrm{N}$ & 24 & 24 \\
\hline
\end{tabular}

** La correlación es significativa al nivel 0,01 (bilateral)

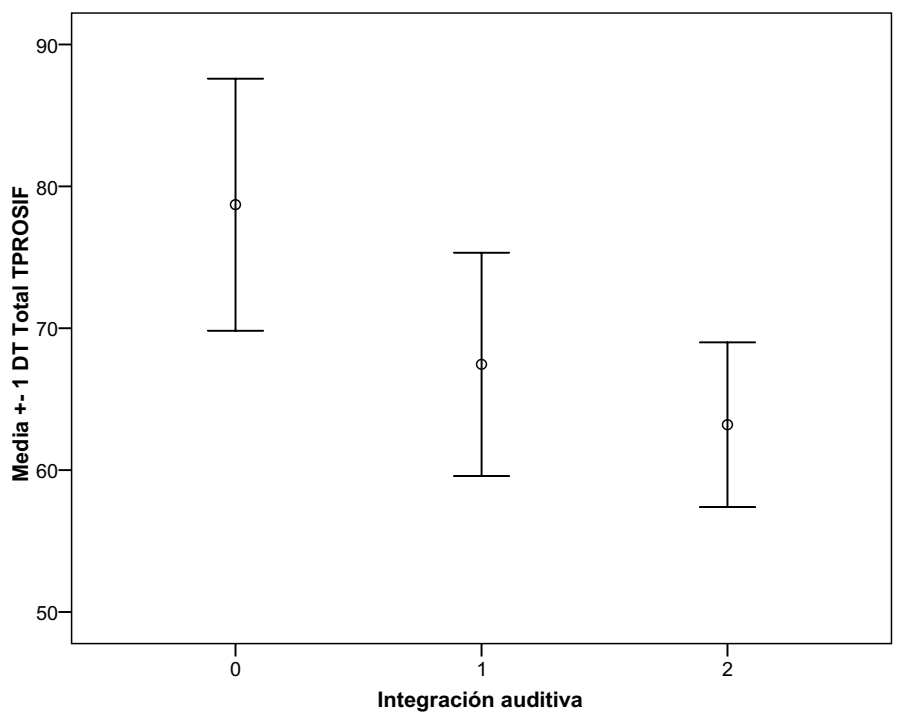

Gráfico 4. Puntaje de TEPROSIF-R y puntaje de la prueba IA. 
Relación entre memoria secuencial auditiva, integración auditiva y desempeño fonológico en niños de 3 años a 3 años 11 meses con trastorno específico del lenguaje de tipo expresivo

Al realizar este análisis en los grupos etarios por separado, observamos en las Tablas 5 y 6 que esta correlación es significativa sólo en el grupo etario de menor edad.

Tabla 5

Correlación entre TEPROSIF-R e IA en grupo etario 3.0-3.5.

\begin{tabular}{|c|c|c|c|c|}
\hline & & & TEPROSIF-R & IA \\
\hline \multirow[t]{6}{*}{ Rho de Spearman } & TEPROSIF & Coeficiente de correlación & 1,0 & $-0,8(* *)$ \\
\hline & & Sig. (bilateral) & . & 0,0 \\
\hline & & $\mathrm{N}$ & 12 & 12 \\
\hline & IA & Coeficiente de correlación & $-0,8(* *)$ & 1,0 \\
\hline & & Sig. (bilateral) & 0,0 & . \\
\hline & & $\mathrm{N}$ & 12 & 12 \\
\hline
\end{tabular}

** La correlación es significativa al nivel 0,01 (bilateral).

Tabla 6

Correlación entre TEPROSIF-R e IA en grupo etario 3.6- 3.11.

\begin{tabular}{|c|c|c|c|c|}
\hline & & & TEPROSIF-R & $\mathrm{IA}$ \\
\hline \multirow[t]{6}{*}{ Rho de Spearman } & TEPROSIF-R & Coeficiente de correlación & 1,0 & $-0,2$ \\
\hline & & Sig. (bilateral) & . & 0,6 \\
\hline & & $\mathrm{N}$ & 12 & 12 \\
\hline & IA & Coeficiente de correlación & $-0,2$ & 1,0 \\
\hline & & Sig. (bilateral) & 0,6 & . \\
\hline & & $\mathrm{N}$ & 12 & 12 \\
\hline
\end{tabular}

Al analizar la correlación entre MSA y TEPROSIF-R, según se presenta la Tabla 7 , se observa que existe una correlación bilateral significativa. Si observamos el Gráfico 5, los datos indican que a menor puntaje obtenido en la prueba de MSA, mayor es el número de PSF emitidos por los participantes en el TEPROSIFR. 
Tabla 7

Correlación entre TEPROSIF-R y MSA.

\begin{tabular}{|c|c|c|c|c|}
\hline & & & TEPROSIF-R & MSA \\
\hline \multirow[t]{6}{*}{ Rho de Spearman } & TEPROSIF-R & Coeficiente de correlación & 1,0 & $-0,7(* *)$ \\
\hline & & Sig. (bilateral) & . & 0,0 \\
\hline & & $\mathrm{N}$ & 24 & 24 \\
\hline & MSA & Coeficiente de correlación & $-0,7(* *)$ & 1,0 \\
\hline & & Sig. (bilateral) & 0,0 & . \\
\hline & & $\mathrm{N}$ & 24 & 24 \\
\hline
\end{tabular}

** La correlación es significativa al nivel 0,01 (bilateral)

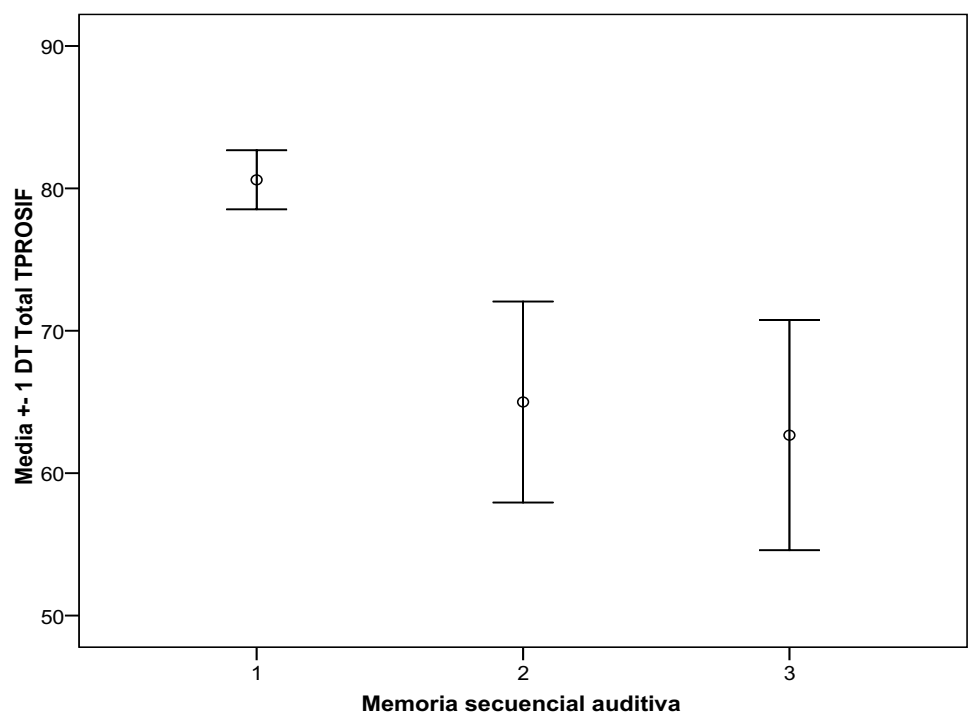

Gráfico 5. Puntaje de TEPROSIF-R y puntaje de MSA.

Al igual que en los resultados anteriores, queda en evidencia en las Tablas 8 y 9, que la correlación entre
MSA y TEPROSIF-R es significativa sólo en el primer grupo etario. 
Tabla 8

Correlación entre TEPROSIF-R MSA en grupo etario 3.0-3.5.

\begin{tabular}{|c|c|c|c|c|}
\hline & & & TEPROSIF-R & MSA \\
\hline \multirow[t]{6}{*}{ Rho de Spearman } & TEPROSIF-R & Coeficiente de correlación & 1,0 & $-0,7\left(^{*}\right)$ \\
\hline & & Sig. (bilateral) & . & 0,0 \\
\hline & & $\mathrm{N}$ & 12 & 12 \\
\hline & MSA & Coeficiente de correlación & $-0,7(*)$ & 1,0 \\
\hline & & Sig. (bilateral) & 0,0 & . \\
\hline & & $\mathrm{N}$ & 12 & 12 \\
\hline
\end{tabular}

* La correlación es significativa al nivel 0,05 (bilateral).

Tabla 9

Correlación entre TEPROSIF-R MSA en grupo etario 3.6- 3.11.

\begin{tabular}{|c|c|c|c|c|}
\hline & & & TEPROSIF-R & MSA \\
\hline \multirow[t]{6}{*}{ Rho de Spearman } & TEPROSIF-R & Coeficiente de correlación & 1,0 & $-0,3$ \\
\hline & & Sig. (bilateral) & . & 0,4 \\
\hline & & $\mathrm{N}$ & 12 & 12 \\
\hline & MSA & Coeficiente de correlación & $-0,3$ & 1,0 \\
\hline & & Sig. (bilateral) & 0,4 & . \\
\hline & & $\mathrm{N}$ & 12 & 12 \\
\hline
\end{tabular}

\section{Discusión}

En el presente estudio se propuso investigar la relación entre el desarrollo de las habilidades psicolingüísticas de MSA e IA con el desempeño fonológico con TEL expresivo divididos en dos grupos etarios: entre 3,0 a 3,5 años y entre 3,6 y 3,11 años. Los resultados evidencian que los niños con TEL expresivo presentan un bajo rendimiento en las pruebas psicolingüísticas y de igual forma un menor desempeño en el test fonológico comparadas con los valores de referencia para su edad. Estos hallazgos concuerdan con el trabajo de Blanco et al. (2008), que informa que los niños con TEL tienen descendidas algunas habilidades psicolingüísticas en comparación con los niños con desarrollo típico del lenguaje. También son consistentes respecto al rendimiento fonológico, con el trabajo de Pavez et al. (2013). Este trabajo mostró que los niños con trastorno fonológico presentan significativamente más PSF que los niños sin dificultades de lenguaje.

Al analizar los resultados obtenidos por cada grupo etario ( 3 años a 3 años 5 meses y 3 años 6 meses a 3 años 11 meses) se observa que en ambas pruebas 
psicolingüísticas los grupos obtuvieron baja puntuación, lo que corresponde a un desempeño no acorde a lo esperado para su edad (ver Tabla 2)., Además, es destacable que el grupo de niños más pequeños obtuvo puntajes más bajos que el grupo de niños mayores (Gráficos 1 y 2).

De la misma forma, en el TEPROSIF-R ambos grupos presentan un rendimiento disminuido que no es acorde a lo que se espera para la edad (ver Tabla 1). Además, se evidencia el mismo patrón que en las pruebas anteriores, ya que el grupo de niños más pequeños obtuvo un menor desempeño que el segundo grupo (ver Gráfico 3). Es importante mencionar que el mayor número de procesos y subprocesos, en niños hispanohablantes, se observa a los 3 y 4 años (Bosch, 2004).

Dado lo anterior, se puede afirmar que los niños con TEL expresivo de 3 años presentan dificultades en tareas psicolingüísticas de IA y MSA, así como también dificultades de producción fonológica, las que disminuyen con la edad.

Respecto al análisis de correlaciones, se observó correlación entre el puntaje del TEPROSIF-R y el puntaje de la prueba de IA. Se encontró una correlación bilateral significativa entre los resultados. Así, a menor puntaje obtenido en la prueba de IA, mayor es el número de PSF emitidos por los participantes en el TEPROSIF-R. Sin embargo, esta correlación es significativa sólo en el grupo etario de menor edad.

Los resultados previos se encuentran en concordancia con lo planteado con Kirk et al. (2009), quienes indican que la IA influye en el proceso de analizar y sintetizar palabras y sílabas, mediante la segmentación y el cierre fonológico. Ello, dado que se demuestra que esta habilidad psicolingüística se relacionada con el desarrollo fonológico a nivel global en niños con TEL expresivo a los 3 años. Además, se observa que esta relación se da principalmente por el grupo de menor edad quienes producen una mayor cantidad de PSF generando un bajo desempeño fonológico.

De igual forma, la relación entre el puntaje del test fonológico y la prueba de MSA, existe una correlación bilateral significativa entre los resultados del TEPROSIF-R y la prueba de MSA. Cabe mencionar que esta relación sería significativa en el grupo etario de menor edad quienes además presentan mayor cantidad de PFS. Nuevamente, los resultados presentados apoyan la propuesta de Kirk et al. (2009), quienes señalan que la MSA es una habilidad utilizada cotidianamente en la producción fonológica y verbal en general. Dado lo anterior, se puede interpretar que el disminuido desempeño en las habilidades de MSA impacta en el desempeño verbal de los niños estudiados, lo que es evidenciado en el disminuido desempeño fonológico registrado en este estudio.

En síntesis, la presente investigación describe cómo influyen en la adquisición y desarrollo fonológico el descenso en IA y MSA en niños de 3 años portadores de TEL expresivo. La evidencia presentada apoya lo expuesto por Maurens et al. (2013) quienes mencionan que es común que estas habilidades se encuentren descendidas en aquellos niños que presentan un trastorno del lenguaje, ya sea asociado a otra patología o de manera específica, como es el caso del TEL. Además, están en concordancia con lo planteado por Triadó y Forns (1989) quienes identifican a estas dos habilidades psicolingüísticas como imprescindibles para un adecuado desarrollo 
del lenguaje.

Los resultados de esta investigación confirman que si estas habilidades se encuentran deficitarias, ello repercute de manera directa en el desempeño del nivel fonológico del lenguaje. Y es que, precisamente al tratar de recordar y analizar una palabra es donde surgiría la base del problema para luego reproducirla de forma correcta.

\section{Conclusiones y Proyección}

En relación con el objetivo del estudio, se puede confirmar que los niños que presentan un bajo desempeño en las habilidades psicolingüísticas de MSA e IA, evidencian también un disminuido desempeño fonológico.

En primer lugar, se puede señalar que el desempeño de los niños en las habilidades de MSA e IA es inferior al desempeño esperado para su edad cronológica en ambos rangos etarios. Esto es más evidente en el rango etario de 3 años a 3 años 5 meses, mientras que en el rango etario de 3 años 6 meses a 3 años 11 meses esta discrepancia es menos notoria.

En segundo lugar, se puede concluir que el desempeño fonológico de los niños participantes del estudio, se comporta de forma esperada según su diagnóstico de TEL expresivo. Es decir, un disminuido rendimiento de este nivel en relación con su edad cronológica tal como señalan los estudios que abordan este diagnóstico.

En tercer lugar, se verifica una correlación entre las variables psicolingüísticas estudiadas y el desempeño fonológico de los niños considerados en su totalidad. Sin embargo, al analizar esta situación por rangos etarios se puede observar que, por una parte, para los niños del rango etario de 3 años a 3 años 5 meses la correlación es directa y estadísticamente significativa tanto para MSA como para IA. Así, se corrobora que en la medida que las habilidades psicolingüísticas disminuyen su desempeño, también se reduce el rendimiento fonológico de los participantes. Por otra parte, para los niños del grupo etario de 3 años 6 meses a 3 años 11 meses, existe igualmente una correlación directa entre las habilidades de MSA e IA y el desempeño fonológico, la que se expresa con menos fuerza sin alcanzar una significación estadística.

Por último, se puede establecer que, desde la perspectiva del número de PSF producidos en ambos rangos etarios, disminuye drásticamente en el segundo grupo etario. Este hecho muestra que en los niños con TEL expresivo estudiados, se observa una aceleración en el desarrollo fonológico durante el trascurso del tercer año de vida. Este fenómeno es similar a lo que se describe para los niños con desarrollo típico del lenguaje, aunque no alcanzan los indicadores de desarrollo fonológico que se describen para la población típica.

En cuanto a las limitaciones del presente estudio, el tamaño muestral es menos de lo que suele evaluarse en estudios de desarrollo típico. Sin embargo, por tratarse de un grupo patológico que presentaba determinadas condiciones de edad y diagnóstico no fue factible obtener mayor número de informantes. Pese a lo descrito, se logró obtener simetría en la conformación de ambos grupos etarios estudiados con 12 sujetos por rango, lo que permitió la aplicación adecuada de pruebas estadísticas para obtener un análisis comparativo. 
Desde la perspectiva de las proyecciones, surge el interés de replicar el estudio con participantes de otros grupos etarios, ya que solo se establecieron los cambios y avances que ocurren dentro de un año de vida. Por lo tanto, sería útil conocer lo qué sucedería entre los 4 y 5 años de edad. Ello permitiría abarcar el periodo que según la literatura resulta crucial para el desarrollo fonológico.

Otra proyección es presentar el análisis de las relaciones existentes entre las habilidades psicolingüísticas y el desempeño fonológico, considerando por separado cada uno de los tipos de PSF estudiados por el TEPROSIF-R.

De la misma forma, sería relevante investigar el efecto de las variables estudiadas en niños con TEL mixto y así conocer y abordar su comportamiento más integralmente. Por otro lado, sería de gran interés proyectar esta investigación en participantes con desarrollo típico del lenguaje, para tener una base de relación y comparación para niños chilenos con desempeños lingüísticos alterados.

Finalmente, este estudio permitirá fundamentar el uso de nuevos métodos evaluativos en el quehacer de los profesionales involucrados en el desarrollo de la lengua, pues este trabajo demuestra la relevancia de incorporar las habilidades psicolingüísticas a la evaluación de niños con TEL.

En síntesis, esta investigación contribuye significativamente en el abordaje terapéutico de los niños con TEL, pues evidencia la influencia de la IA y la MSA en el disminuido desempeño fonológico de los niños estudiados. Por lo tanto, son habilidades que deberían incluirse en los objetivos terapéuticos y en el plan de intervención que se realiza para superar las dificultades lingüísticas de los niños con TEL.
Agradecimiento: trabajo enmarcado en el Proyecto DINR 03/2017.

\section{Referencias}

Blanco, S., González, F., Ramírez, F., Torres, C., \& Valenzuela, M. (2008). Habilidades Psicolingüísticas en niños con Trastorno Específico del Lenguaje de Kínder y nivel básico 1 (Seminario de Licenciatura, Universidad de Chile). Recuperado de http://www.tesis.uchile.cl/tesis/uchile/2008/blanco_s/sou rces/blanco_s.pdf

Bosch, L. (2003). Trastornos del desarrollo fonético y fonológico. En M. Rondal (Ed.), Manual de desarrollo y alteraciones del lenguaje (pp. 189-204). Barcelona: Masson.

Bosch, L. (2004). Evaluacion fonologica del habla infantil. Recuperado de https://books.google.cl/books?id=JKKr5anu50YC\&printsec $=$ frontcover $\& \mathrm{dq}=\% 22$ Evaluaci\%C3\%B3n+fonol\%C3\%B3gica +del+habla+infantil\%22\&hl=es419\&sa=X\&ved=0ahUKEwiqz2g64XmAhWEC9QKHQ_aDkcQ6AEIKTAA\#v=onepage\&q=\% 22Evaluaci\%C3\%B3n\%20fonol\%C3\%B3gica\%20del\%20habl $a \% 20$ infantil\%22\&f=false

Carballo, G. (2012). Guía para la evaluación del TEL: Algunas consideraciones. Revista de Logopedia, Foniatría y Audiología, 32(2), 87-93. https://doi.org/10.1016/j.rlfa.2012.03.004

Ingram, D. (1983). Trastornos fonológicos en el niño. Barcelona: Médica y Técnica.

Kirk, S. A., MacCarthy, J. J., \& Kirk, W. D. (2009). ITPA, Test Illinois de Aptitudes Psicolingüísticas. Madrid: TEA Ediciones, S.A.

Maurens, N., Olivares, F., \& Vergara, V. (2013). Habilidades psicolinguisticas en escolares de entre 6 años y 9 años 11 meses con trastorno por deficit atencional e hiperactividad (Seminario de Licenciatura, Universidad de Chile). Recuperado

de

http://repositorio.uchile.cl/handle/2250/116722

Pavez, M. M. (1990). Procesos fonológicos de simplificación en niños: Su incidencia en los trastornos del lenguaje. RLA: Revista de Lingüística Teórica y Aplicada, (28), 123-134. Recuperado

de 
https://dialnet.unirioja.es/servlet/articulo?codigo=16325

Pavez, M. M., Coloma, C. J., Maggiolo, M., \& Peñaloza, C. (2013). Procesos de simplificación fonológica en niños de 4, 5 y 6 años con dificultades fonológicas. Revista Chilena de Fonoaudiología, 12 , 49-61. https://doi.org/10.5354/rcdf.v12i0.29926

Pavez, M. M., Maggiolo, M., \& Coloma, C. J. (2008). Test para evaluar procesos de simplificación fonológica: TEPROSIF-R. Santiago, Chile: Ediciones UC.

Pavez, M. M., Maggiolo, M., Peñaloza, C., \& Coloma, C. J. (2009). Desarrollo fonológico en niños de 3 a 6 años: Incidencia de la edad, el género y el nivel socioeconómico. RLA: Revista de Lingüística Teórica y Aplicada, 47(2), 89$109 . \quad$ Recuperado de https://dialnet.unirioja.es/servlet/articulo?codigo=313009 7

Stampe, D. (1969). The acquisition of phonetic representation. 433-444. Chicago: Linguistic Society.

Triadó, C., \& Forns, M. (1989). La evaluación del lenguaje: Una aproximación evolutiva. España: Anthropos Editorial. 